\title{
Intravascular Images of Coronary Stenosis with Multiple Channels in a Patient with Antiphospholipid Syndrome: The Optical Coherence Tomography Findings
}

\author{
Shigenori Ito and Takahiro Hasuo
}

\begin{abstract}
Intravascular images of coronary stenosis by antiphospholipid syndrome (APS) would be beneficial to understand the mechanism of this disease. A 59-year-old woman with APS/systemic lupus erythematosis (SLE) underwent emergent coronary angiography which revealed stenosis with micro channels in the proximal left anterior descending artery. According to optical coherence tomography (OCT), the central lumen was surrounded by a thick septum with a homogenous and high intensity. Multiple small channels existed near the vessel wall with diffuse intimal hyperplasia. White thrombi were floating distal to the stenosis. Intravascular images obtained by optical coherence tomography (OCT) revealed the microstructure of complex coronary stenosis, which had ambiguous findings on angiography and intravascular ultrasound (IVUS) in an acute coronary syndrome (ACS) patient with APS/SLE.
\end{abstract}

Key words: antiphospholipid syndrome, systemic lupus erythematosis, optical coherence tomography, recanalized thrombus, acute coronary syndrome

(Intern Med 56: 1351-1356, 2017)

(DOI: 10.2169/internalmedicine.56.7804)

\section{Introduction}

It is reported that the prevalence rates of myocardial infarction and angina pectoris in patients with antiphospholipid syndrome (APS) are 5.5\% and $2.7 \%$, respectively (1). Although the incidence of ischemic heart disease is not high compared with that of cerebrovascular and/or deep vein thrombosis, myocardial infarction is an important complication and several case reports have shown acute myocardial infarction to occur in young patients with primary APS, but without any coronary risk factors (2-4) and APS associated with systemic lupus erythematosis (SLE) (5). Besides such thrombotic events, atherosclerosis has also been reported to occur prematurely in patients with SLE, independent of any traditional risk factors for cardiovascular disease (6). Furthermore, in patients with SLE, the prevalence of coronaryartery atherosclerosis is elevated and the age at onset is reduced according to the study using electron-beam computed tomography to screen for the presence of coronary-artery calcification (7). The relationship between thrombosis and atheroscrelosis in the coronary arteries has not yet been adequately reported in patients with APS and/or SLE.

In our case, the image characteristics of coronary stenosis in a patient with APS and SLE manifesting acute coronary syndrome (ACS) could be evaluated by intravascular ultrasound (IVUS) and optical coherence tomography (OCT).

\section{Case Report}

A 59-year-old woman was admitted to our hospital because of the repeated attacks of left shoulder pain which were sometimes associated with cold sweat lasting for 30 seconds over the previous two-week period. Her past history included SLE, cerebral infarction, deep vein thrombosis. Her coronary risk factors were hypertension, dyslipidemia, and being a current smoker. Medical treatments included warfarin, predonisolon, statin, and angiotensin receptor blocker. No antiplatelet agent had been given. The electrocardiogram findings on admission showed $\mathrm{T}$ wave inversion in leads $\mathrm{I}$, 

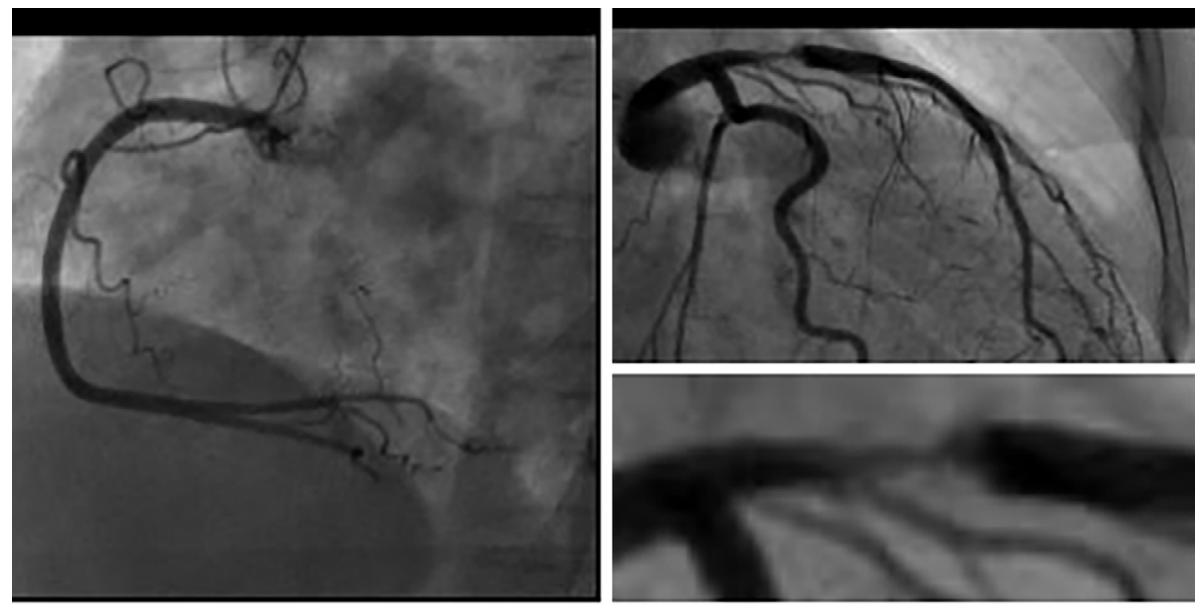

A
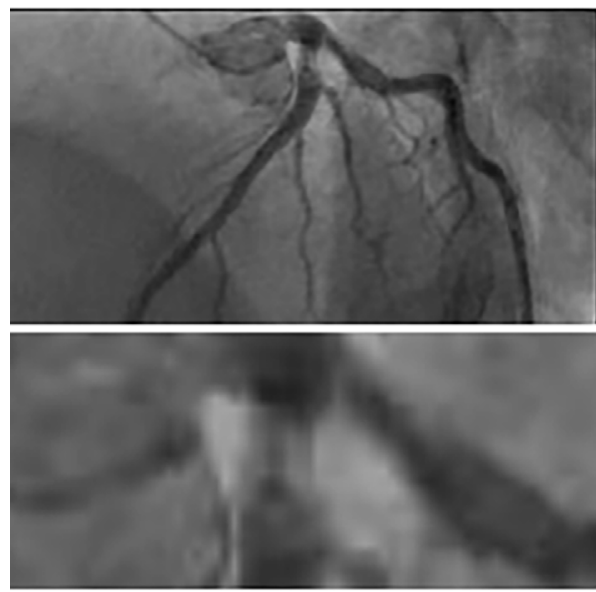

C

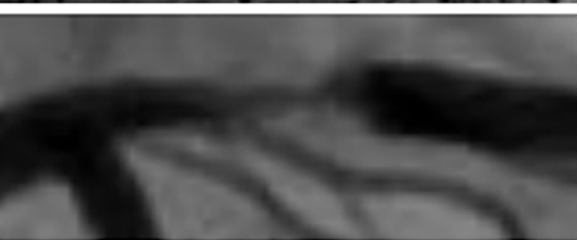

B
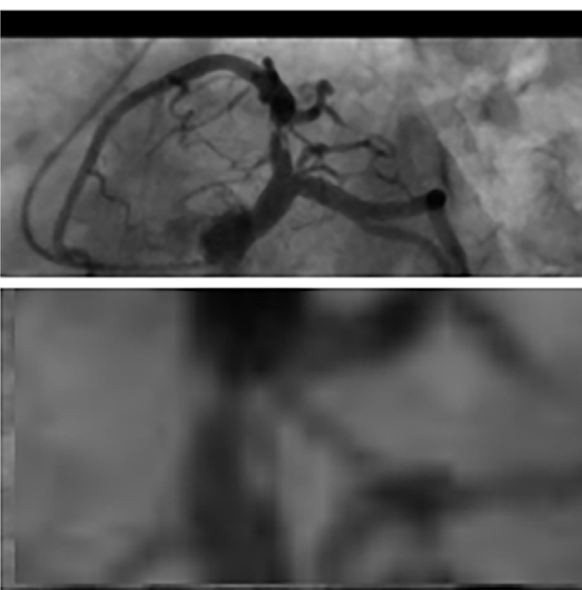

D

Figure 1. Baseline coronary angiography. Right coronary artery (A: LAO view). No atheroscrelotic lesions are observed. Left coronary artery (B: RAO caudal view, C: LAO cranial view, D: LAO caudal view). Tubular stenosis can be seen in the proximal LAD.This lesion has two linear contrast defects, which divide the lumen into a central lumen and lateral multiple small channels. There are no other atherosclerotic lesions in the distal LAD, LCx, and the RCA. Magnified pictures in the lower panels of $\mathrm{B}, \mathrm{C}$, and $\mathrm{D}$ reveal similar finding for this lesion. LAO: left anterior oblique, RAO: right anterior oblique, LAD: left anterior descending artery, LCx: left circumflex artery

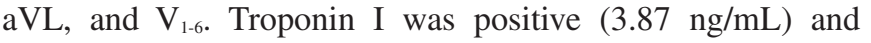
creatine phosphokinase was not elevated. The anti $\beta 2$ glycoprotein antibody I level was $\geq 125 \mathrm{U} / \mathrm{mL}$ (normal range $\leq$ $3.5 \mathrm{U} / \mathrm{mL}$ ) and the anti cardiolipin antibody level was $\geq 120$ $\mathrm{U} / \mathrm{mL}$ (normal range $\leq 10 \mathrm{U} / \mathrm{mL}$ ). She was diagnosed to have acute coronary syndrome and therefore underwent emergent coronary angiography (Fig. 1), which revealed severe stenosis with lateral small channels in the proximal left anterior descending artery (LAD). Angiographically detectable atheroscrelotic lesions were not found in either the left circumflex artery (LCx) or the right coronary artery (RCA). Percutaneous coronary intervention (PCI) was performed for the LAD lesion. Before PCI she underwent OCT (Fig. 2) and IVUS (Fig. 3) because of unusual angiographic findings (Fig. 1). According to OCT, a multiple lumen structure was clearly detected, which was separated by the septum with a homogenous high intensity. Surrounding the central lumen, multiple small channels existed near the vessel wall, which demonstrated intimal hyperplasia. Although this lesion showed a so called honeycomb- or lotus root-like appearance, the inter luminal septum was circular and thick in our case (Fig. 2A and B). White thrombi were floating distal to the stenosis (Fig. 2B). This finding is consistent with acute coronary syndrome. A longitudinal section revealed the whole structure of the stenotic lesion and white thrombus (Fig. 2C). IVUS showed a donut-shaped material with broad band and heterogeneous echogeneity in the lesion (Fig. 3). A circular narrow low echoic space, possibly with a blood flow, was detected outside this material. Distal protection with Filtrap ${ }^{\mathrm{TM}}$ was performed before stent implantation. Filter no reflow occurred after balloon dilatation. No material was aspirated. After pre dilatation with a Lacrosse NSE 


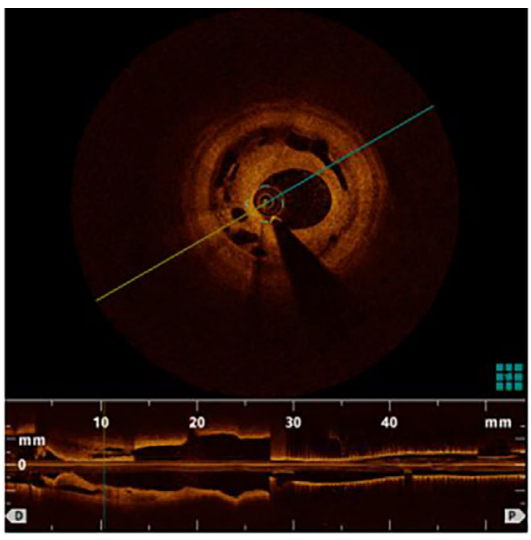

A

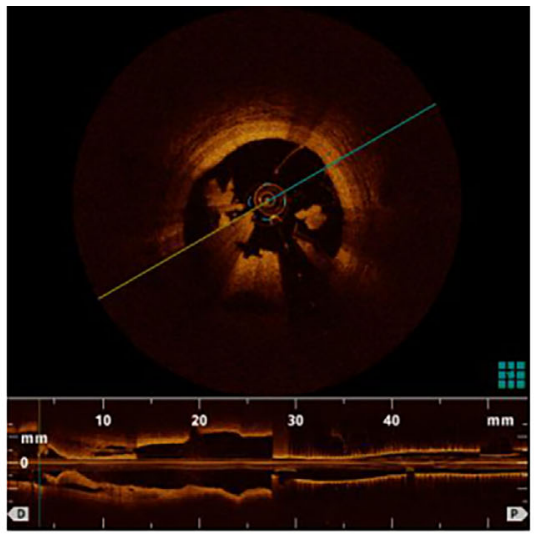

B

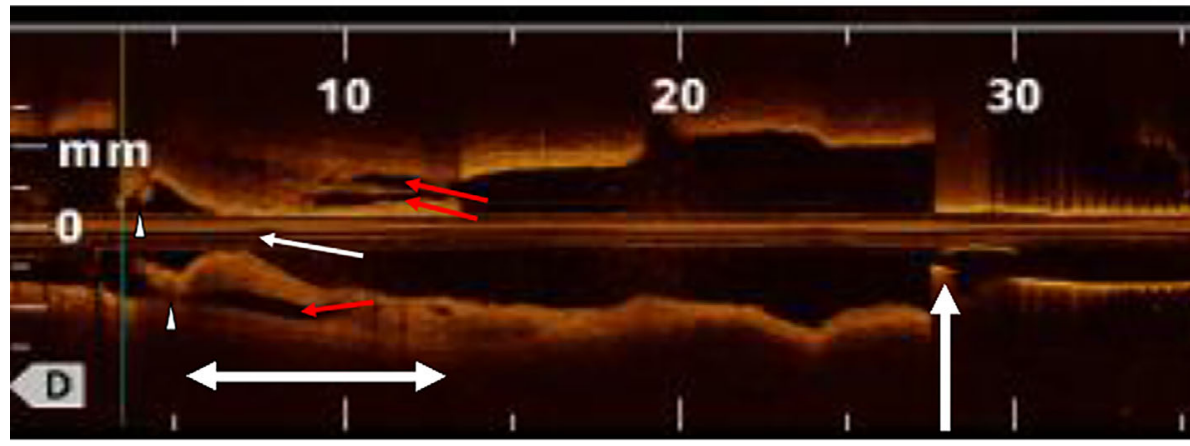

stenosis in the proximal LAD

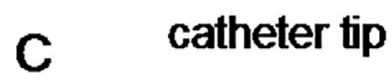

Figure 2. Optical coherence tomography before PCI. In the stenosis, a central lumen and circumferential small channels separated by thick high intensity septum are detected (A). Floating white thrombus can be detected just distal to the stenosis $(B)$. The whole structure of this lesion can be detected by longitudinal section of optical coherence tomography $(C)$. A white arrow indicates the central lumen. The red arrows indicate the multiple small channels surrounding the central lumen. Two white arrow heads show white thrombi.

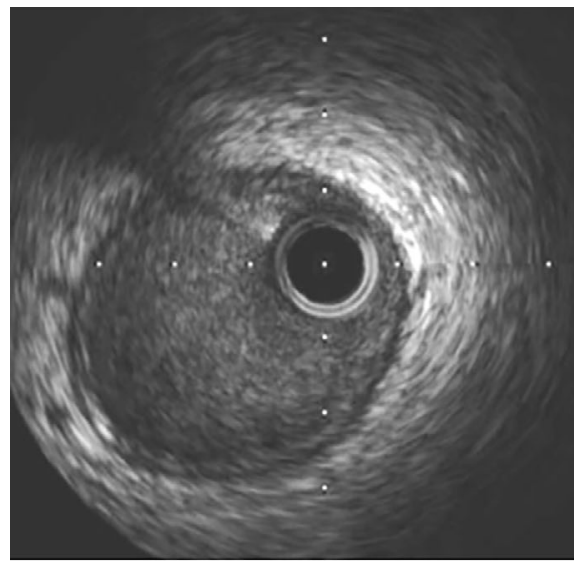

A

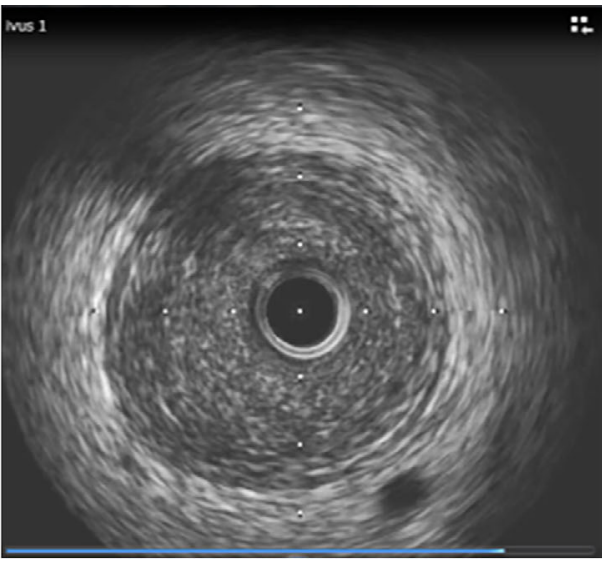

B

Figure 3. Intravascular ultrasound tomography. The proximal reference vessel has little intimal hyperplasia (A). The stenotic lesion (B) shows the presence of a donut-shaped material with heterogeneous echogeneity. A circular narrow low echoic space is detected outside of this material.

scoring balloon ${ }^{\mathrm{TM}}(3.5 \times 13 \mathrm{~mm})$, a Multilink $8^{\mathrm{TM}}$ stent $(4.0 \times$ $23 \mathrm{~mm}$ ) was deployed. Thrombolysis in the myocardial infarction (TIMI) 3 coronary flow could be achieved after Filtrap retrieval (Fig. 4). The patient was treated with dual anti- platelet therapy (aspirin and clopidogrel) in addition to warfarin after PCI. Follow-up coronary angiography at six months showed no restenosis and OCT revealed diffuse instent intimal hyperplasia (Fig. 5). The acetylcholine provo- 


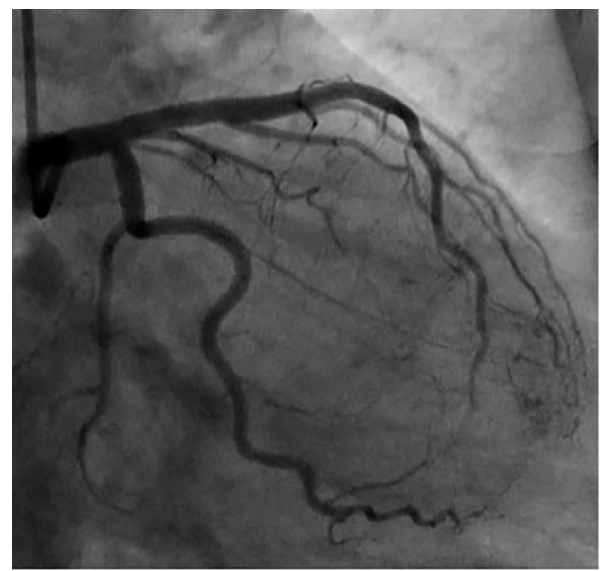

A

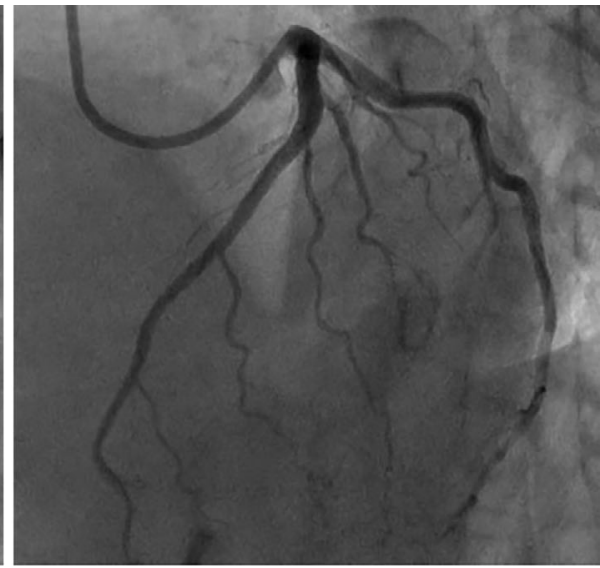

B

Figure 4. Final coronary angiography after PCI. Excellent results were obtained. Neither any residual stenosis nor thrombus was found in the RAO caudal (A) and the LAO cranial (B) views. PCI: percutaneous coronary intervention, RAO: right anterior oblique, LAO: left anterior oblique

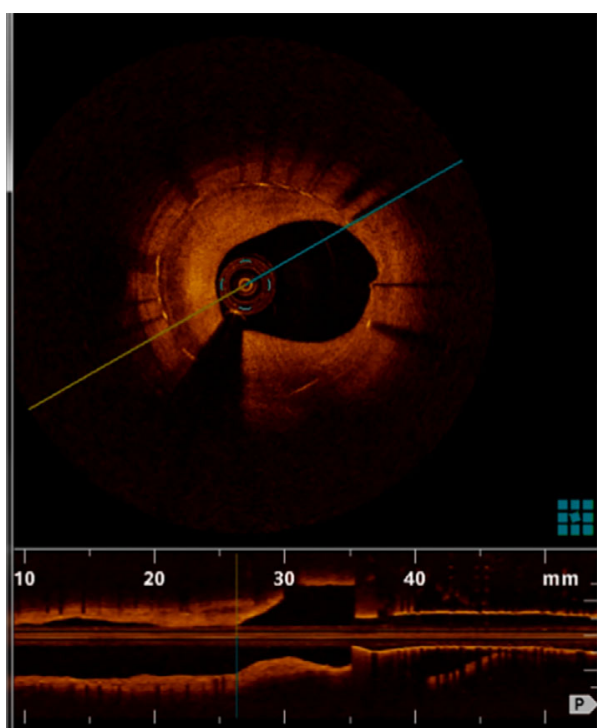

Figure 5. Optical coherence tomography at the six-month follow-up. Although restenosis was not detected by angiography, significant amount of intimal hyperplasia could be detected.

cation test induced coronary spasm in the reference vessel distal to the implanted stent.

\section{Discussion}

To the best of our knowledge, this is the first report which shows the intravascular images by IVUS and OCT for coronary stenosis with lateral small channels in a patient with APS and SLE. Although several case reports have already shown acute myocardial infarction to occur in young patients with primary APS but without any coronary risk factors (2-4) and APS associated with SLE (5), these reports did not describe the intravascular image characteristics of coronary stenosis and/or thrombus. In our ACS case, OCT revealed a multi lumen structure, suggesting a recanalized thrombus, on the intimal hyperplasia with white thrombus distal to the stenosis.

\section{Mechanism of stenosis formation and thrombus}

In patients with APS, coronary thrombosis has been reported to occur even in those who did not have any coronary risk factors or coronary atherosclerosis (2, 5, 8-10). The pathogenesis could be the thrombus formation based on the prothrombotic state caused by several pathways, such as the activation of endothelial cells (activation of adhesion molecules or tissue factor), the activation of thrombocytes (induction of thromboxane A2, increased adhesion), the activation of coagulation cascade, and the inhibition of anticoagulation (inhibition of plasminogen/plasmin, tissue plasminogen activator, and protein $\mathrm{C}$ and $\mathrm{S}$ ) etc. (11). Atherosclerosis also occurs prematurely in patients with SLE, independent of any traditional risk factors for cardiovascular disease (6). In our case, the combination of APS/SLE and coronary risk factors might have induced the occurrence of coronary endothelial dysfunction and atheroscrelosis (intimal hyperplasia) simultaneously. Furthermore, coronary spasm was also considered as a potential cause of thrombosis (12-15), because the symptoms included repeated episodes of rest angina and a provoked coronary spasm was found by the spasm provocation test with intracoronary acetylcholine administration at follow-up coronary angiography. A previously formed thrombus might have partially recanalized to form the present stenosis with a complex structure. Finally, the organic stenosis and spasm might have reduced the coronary blood flow leading to the onset of acute coronary syndrome without any coronary plaque rupture.

\section{Evaluation of imaging characteristics of the stenosis}

Our case may have demonstrated a honeycomb/lotus root appearance (16-20) because it had multiple channels. However, the following characteristics distinguish our case from previously reported OCT images of multiple channels struc- 
ture. Our case demonstrated a thick septum and intimal hyperplasia. The multiple lumens were composed of a central lumen and small channels surrounding the central lumen rather than similar multiple lumens. The accumulation of OCT images of coronary stenosis/thrombus in patients with APS is thus necessary to determine whether or not these characteristic are peculiar to APS and/or SLE patients. IVUS could not demonstrate any specific findings that OCT could not provide.

\section{Restenosis after PCI and APS}

The acute effects of PCI [balloon angioplasty $(21,22)$ or stent implantation $(3,8,16)$ ] have been reported as a treatment strategy for acute myocardial infarction/ACS in patients with APS and/or SLE. In contrast, an increased risk of restenosis in patients with APS who undergo PCI has been previously reported in a few small studies. Gurlek et al. (23) reported a strong association between the levels of anticardiolipin antibodies and the outcome of PCI after an ACS. In this study, higher levels of anticardiolipin antibodies predicted increased rates of restenosis after PCI, without any effect on mortality. Similarly, patients with APS who undergo PCI have worse long-term clinical outcomes, driven by higher rates of revascularization, than other patients undergoing PCI despite the similar use of drug-eluting stents and a similar diabetes status as that of the control groups (24).

Thus, the use of a drug-eluting stent in our case might have been an alternative strategy according to these reports. However, we selected a bare metal stent rather than a drugeluting stent, because we thought the vessel was large enough to expect a low restenosis rate and dual antiplatelet therapy could be stopped immediately in the case of any bleeding complications. Our patient did not show any restenosis after bare metal stent implantation at follow-up coronary angiography six months later. Meanwhile, an OCT follow-up revealed a considerable amount of in-stent intimal hyperplasia. It is therefore necessary to pay meticulous attention to this patient during a further long-term follow-up.

Intravascular images obtained by OCT was thus found to clearly demonstrate the microstructure of complex coronary stenosis in an ACS patient with APS and SLE.

The authors state that they have no Conflict of Interest (COI).

\section{References}

1. Cervera R, Piette JC, Font J, et al. Antiphospholipid syndrome: clinical and immunologic manifestations and patterns of disease expression in a cohort of 1,000 patients. Arthritis Rheum 46: 1019-1027, 2002.

2. Jurado OM, Duran J, Martinez A, Castellon JM, Gutierrez MA. Acute myocardial infarction in a man without coronary atheromatosis and antiphospholipid syndrome: report of one case. Rev Med Chil 137: 1478-1481, 2009.

3. Biceroglu S, Ildizli Demirbas M, Karaca M, Yalcin M, Yilmaz H. Acute thrombotic occlusion of right coronary and left circumflex coronary arteries in a patient with antiphospholipid syndrome: successful stent implantation. Case Rep Med 2010: 198594, 2010.

4. Grzybczak R, Undas A, Rostoff P, et al. Life-threatening cardiac manifestations of primary antiphospholipid syndrome. Heart Vessels 25: 267-269, 2010.

5. Zhang B, Jiang DM, Zhou XC, Qi GX. Myocardial infarction in a patient with systemic lupus erythematosus and antiphospholipid syndrome. Chin Med J (Engl) 124: 2392-2395, 2011.

6. Roman MJ, Shanker BA, Davis A, et al. Prevalence and correlates of accelerated atherosclerosis in systemic lupus erythematosus. N Engl J Med 349: 2399-2406, 2003.

7. Asanuma Y, Oeser A, Shintani AK, et al. Premature coronaryartery atherosclerosis in systemic lupus erythematosus. N Engl J Med 349: 2407-2415, 2003.

8. Abid L, Frikha F, Bahloul Z, Kammoun S. Acute myocardial infarction in young adults with antiphospholipid syndrome: Report of two cases and literature review. Pan Afr Med J 8: 13, 2011.

9. Maor E, Fefer P, Varon D, et al. Thrombophilic state in young patients with acute myocardial infarction. J Thromb Thrombolysis 39: 474-480, 2015.

10. Vergallo R, Aguirre AD, Abtahian F, et al. Recurrent myocardial infarctions and premature coronary atherosclerosis in a 23 -year-old man with antiphospholipid syndrome. Thromb Haemost 115: 237239, 2016.

11. Koniari I, Siminelakis SN, Baikoussis NG, Papadopoulos G, Goudevenos J, Apostolakis E. Antiphospholipid syndrome; its implication in cardiovascular diseases: A review. J Cardiothorac Surg 5: 101, 2010.

12. Oshima S, Yasue H, Ogawa H, Okumura K, Matsuyama K. Fibrinopeptide a is released into the coronary circulation after coronary spasm. Circulation 82: 2222-2225, 1990.

13. Misumi I, Ogawa H, Masuda T, Sakamoto T, Okumura K, Yasue $\mathrm{H}$. Increased plasma plasminogen activator inhibitor activity after coronary spasm. Int J Cardiol 41: 21-29, 1993.

14. Miyamoto S, Ogawa H, Soejima H, et al. Formation of platelet aggregates after attacks of coronary spastic angina pectoris. Am J Cardiol 85: 494-497, A10, 2000.

15. Miyamoto S, Ogawa H, Soejima H, et al. Enhanced platelet aggregation in the coronary circulation after coronary spasm. Thromb Res 103: 377-386, 2001.

16. Nakanishi T, Kawata M, Matsuura $T$, et al. A case of coronary lesion with lotus root appearance treated by percutaneous coronary intervention with intravascular ultrasound guidance. Cardiovasc Interv Ther 25: 131-134, 2010.

17. Kang SJ, Nakano M, Virmani R, et al. Oct findings in patients with recanalization of organized thrombi in coronary arteries. JACC Cardiovasc Imaging 5: 725-732, 2012.

18. Musashi M, Tada N, Uemura N, et al. Multivessel honeycomb-like structure finding in optical coherence tomography. JACC Cardiovasc Interv 7: e7-e8, 2014.

19. Kimura $T$, Itoh $T$, Fusazaki $T$, Nakamura $M$, Morino $Y$. A honeycomb-like structure in the right coronary artery visualized by three-dimensional optical coherence tomography. Coron Artery Dis 26: $356-360,2015$

20. Suzuki M, Seki A, Nishikawa K, Takamisawa I, Tobaru T, Sumiyoshi T. Novel physiological insight into a lotus root appearance in stable coronary artery diseases; report of two cases. Cardiovasc Interv Ther 31: 128-130, 2016.

21. Takeuchi S, Obayashi T, Toyama J. Primary antiphospholipid syndrome with acute myocardial infarction recanalised by ptca. Heart 79: 96-98, 1998.

22. Harada T, Nakamura F, Ikeda Y, et al. Successful catheter intervention for acute coronary syndrome in a patient with antiphospholipid syndrome. Jpn Heart J 42: 627-631, 2001.

23. Gurlek A, Ozdol C, Pamir G, Dincer I, Tutkak H, Oral D. Association between anticardiolipin antibodies and recurrent cardiac 
Intern Med 56: 1351-1356, 2017 DOI: 10.2169/internalmedicine.56.7804

events in patients with acute coronary syndrome. Int Heart $\mathbf{J} \mathbf{4 6}$ : 631-638, 2005.

24. Perl L, Netzer A, Rechavia E, et al. Long-term outcome of patients with antiphospholipid syndrome who undergo percutaneous coronary intervention. Cardiology 122: 76-82, 2012.
The Internal Medicine is an Open Access article distributed under the Creative Commons Attribution-NonCommercial-NoDerivatives 4.0 International License. To view the details of this license, please visit (https://creativecommons.org/licenses/ by-nc-nd/4.0/).

(C) 2017 The Japanese Society of Internal Medicine http://www.naika.or.jp/imonline/index.html 